OPEN ACCESS

Edited by:

Yong Qin,

The University of Texas at El Paso,

United States

Reviewed by:

Camelia Quek,

Melanoma Institute Australia, Australia

Olga Vornicova,

Ha'Emek Medical Center, Israel

${ }^{*}$ Correspondence:

Changchun Zeng

zengchch@glmc.edu.cn

Specialty section:

This article was submitted to

Skin Cancer,

a section of the journal

Frontiers in Oncology

Received: 13 September 2021 Accepted: 22 October 2021

Published: 04 November 2021

Citation:

Zeng H, Liu F, Zhou H and Zeng C (2021) Individualized Treatment

Strategy for Cutaneous

Melanoma: Where Are We Now and Where Are We Going?

Front. Oncol. 11:775100.

doi: 10.3389/fonc.2021.775100

\section{Individualized Treatment Strategy for Cutaneous Melanoma: Where Are We Now and Where Are We Going?}

\author{
Huihua Zeng ${ }^{1}$, Fen Liu ${ }^{2}$, Hairong Zhou ${ }^{1}$ and Changchun Zeng ${ }^{3 *}$ \\ ${ }^{1}$ Department of General Medicine, Shenzhen Longhua District Central Hospital, Shenzhen, China, ${ }^{2}$ Department of Chinese \\ Medicine, Shenzhen Longhua District Central Hospital, Shenzhen, China, ${ }^{3}$ Department of Medical Laboratory, Shenzhen \\ Longhua District Central Hospital, Guangdong Medical University, Shenzhen, China
}

In the past several decades, innovative research in cancer biology and immunology has contributed to novel therapeutics, such as targeted therapy and immunotherapy, which have transformed the management of patients with melanoma. Despite the remarkable therapeutic outcomes of targeted treatments targeting MAPK signaling and immunotherapy that suppresses immune checkpoints, some individuals acquire therapeutic resistance and disease recurrence. This review summarizes the current understanding of melanoma genetic variations and discusses individualized melanoma therapy options, particularly for advanced or metastatic melanoma, as well as potential drug resistance mechanisms. A deeper understanding of individualized treatment will assist in improving clinical outcomes for patients with cutaneous melanoma.

Keywords: MAPK, melanoma, targeted therapy, immunotherapy, resistance

\section{INTRODUCTION}

Currently, melanoma is the most common type of skin cancer. Despite accounting for approximately $1 \%$ of skin malignancies, melanoma is responsible for most skin cancer-related mortality. GLOBOCAN 2020 estimates that 324,635 new skin melanoma cases and 57,043 new melanoma of skin deaths occurred in 2020 (1). In 2021, approximately 101,280 new skin melanoma cases are predicted in the United States. The incidence of skin melanoma continues to rise, but it has begun to decline in recent birth cohorts. From 2009 to 2013, the melanoma mortality rate held steady but then dropped by $5.7 \%$ yearly for the following five years. Recently, metastatic melanoma death rates have been steadily declining, possibly owing to advances in therapy. The 5 -year survival rate for early-stage melanoma is $99 \%$, but just $27 \%$ for metastatic melanoma. When melanoma is detected early, it is feasible to treat it surgically, resulting in increased survival rates. However, therapeutic interventions become restricted if the melanoma has progressed to distant organs (2).

Primary melanoma is linked to a variety of risk factors, including male sex, old age ( $>60$ years), phenotypic predisposition (atypical mole or dysplastic nevus pattern, increased mole count, sunphenotype or tendency to sunburn, red hair-blue eyes or Fitzpatrick skin type I or pheomelanin predominant phenotype), personal medical history or comorbidities (multiple or blistering sunburns, actinic keratosis or non-melanoma skin cancer, childhood cancer, solid organ transplantation, hematopoietic cell transplantation, human immunodeficiency virus or acquired 
immunodeficiency syndrome, xeroderma pigmentosum), environmental factors (tanning bed use, residence in sunnier climate or latitude nearer to equator, intermittent, intense sun exposure, chronic sun exposure), and genetic predisposition (family history of cutaneous melanoma, pancreatic, renal and/ or breast cancer, astrocytoma, uveal melanoma, and/or mesothelioma, germline mutation, including CDKN2A, CDK4, MC1R, BRCA2, BAP1, TERT, MITF, and PTEN) (3-7).

BRAF, MEK, NRAS, HRAS, KRAS, c-KIT, c-Met, VEGFR, PTEN, and PIK3CA mutations are common in melanoma. Activation of Ras/Raf/MAPK, PI3K/AKT/mTOR, and JAK/STAT signaling pathways leads to malignant phenotypes and tumorigenesis $(8,9)$. Advances in the study of biological functions and molecular mechanisms may provide potential therapeutic targets for patients with melanoma. The revolutionary discoveries of targeted therapy and immunotherapy, two highly effective treatment strategies, profoundly altered the standard of practice for melanoma, leading to a renewed hope of preventing the disease (10). Unfortunately, not all patients respond to targeted or immunotherapy. Moreover, most patients eventually develop drug resistance and lack treatment options. Therefore, research into resistance mechanisms and novel treatment alternatives remains critical. Here, we present an overview of treatment options and future perspectives in melanoma.

\section{GENETIC ALTERATIONS IN MELANOMA}

Specific genetic alterations regulate the onset and development of melanoma. The common deleterious mutations closely associated with melanoma were identified (Table 1). The functional mutation burden of BRAF, CDKN2A, MAP2K1, NRAS, PTEN, TP53, PPP6C, RAC1, SNX31, TACC1 and STK19 was statistically significant. Deletions of PTEN and CDKN2A and gains of CCND1, MITF, and TERT, were identified in somatic copy-number alterations profiles. $83 \%$ $(100 / 121)$ of melanoma cases had highly recurrent mutations in NRAS $(27 / 121)$ or BRAF $(n=73 / 121)$ that were mutually exclusive. Highly recurrent mutations in the BRAF V600 codon, which accounts for 35-50\% of melanoma, and the Q61 codon, which accounts for $10-25 \%$ of melanoma, contribute to the evolution of highly selective kinase inhibitors for the MAPK pathway (9). 13 significantly mutated genes (SMGs), including BRAF, NRAS, CDKN2A, TP53, PTEN, RAC1, MAP2K1, PPP6C, and ARID2 were identified using functional mutation burden and loss-of-function tests. UV-induced highly recurrent alterations were identified in SMGs, including IDH1 (6.2\%) and RAC1 (6.9\%). In addition, abnormal activation of RAS/ MAPK/AKT, cell cycle, and apoptosis pathway occurred in $91 \%$, $69 \%$, and $19 \%$ of samples, respectively. According to the pattern

TABLE 1 | Molecular alterations in melanoma.

\begin{tabular}{|c|c|c|c|c|c|}
\hline Gene & Mutation & Locus & Freq (\%) & Pathway & Reference \\
\hline CCND1 & Mut; amp & $11 q 13$ & 5 & Cell-cycle pathway & $(9,11,12)$ \\
\hline CDK4 & Mut; amp & $12 q 14$ & 5 & Cell-cycle pathway & $(9,11,12)$ \\
\hline CDKN2A & Mut; del; hm & $9 p 21$ & 29 & Cell-cycle pathway & $(9,11,12)$ \\
\hline CDKN2B & Mut; del; hm & $9 p 21$ & 18 & Cell-cycle pathway & $(9,11,12)$ \\
\hline RB1 & Mut & $13 q 14.2$ & 5 & Cell-cycle pathway & $(9,11,12)$ \\
\hline TP53 & Mut & $17 p 13.1$ & 14 & DNA damage response and cell death pathways & $(9,11,12)$ \\
\hline ARID2 & Mut & $12 q 12$ & 13 & Epigenetics & $(9,11,12)$ \\
\hline $\mathrm{EZH} 2$ & Mut & $7 q 35-q 36$ & 7 & Epigenetics & $(9,11,12)$ \\
\hline $\mathrm{IDH} 1$ & Mut & 2q33.3 & 5 & Epigenetics & $(9,11,12)$ \\
\hline BRAF & Mut; amp; fusion & $7 q 34$ & 49 & MAPK pathway & $(9,11,12)$ \\
\hline GNA11 & Mut & 19p13.3 & 3 & MAPK pathway & $(9,11,12)$ \\
\hline GNAQ & Mut & $9 q 21$ & 2.2 & MAPK pathway & $(9,11,12)$ \\
\hline HRAS & Mut & $11 p 15.5$ & 1.3 & MAPK pathway & $(9,11,12)$ \\
\hline KDR & Mut; amp & $4 q 11-q 12$ & 13 & MAPK pathway & $(9,11,12)$ \\
\hline $\mathrm{KIT}$ & Mut; amp & $4 q 12$ & 6 & MAPK pathway & $(9,11,12)$ \\
\hline KRAS & Mut & $12 p 12.1$ & 2.2 & MAPK pathway & $(9,11,12)$ \\
\hline MAP2K1 & Mut; amp & $15 q 22.31$ & 6 & MAPK pathway & $(9,11,12)$ \\
\hline MTOR & Mut & $1 p 36.2$ & 9 & MAPK pathway & $(9,11,12)$ \\
\hline NF1 & Mut & $17 q 11.2$ & 13 & MAPK pathway & $(9,11,12)$ \\
\hline NRAS & Mut; amp & 1p13.2 & 24 & MAPK pathway & $(9,11,12)$ \\
\hline PDGFRA & Mut; amp & $4 q 12$ & 9 & MAPK pathway & $(9,11,12)$ \\
\hline RAC1 & Mut; amp & $7 p 22$ & 7 & MAPK pathway & $(9,11,12)$ \\
\hline AKT3 & Mut; amp; fusion & $1 q 44$ & 5 & PI3K/Akt/mTOR Pathway & $(9,11,12)$ \\
\hline PIK3CA & Mut & $3 q 26.3$ & 4 & PI3K/Akt/mTOR Pathway & $(9,11,12)$ \\
\hline PTEN & Mut; del & $10 \mathrm{q} 23.3$ & 13 & PI3K/Akt/mTOR Pathway & $(9,11,12)$ \\
\hline TSC1 & Mut; amp & $9 q 34$ & 4 & PI3K/Akt/mTOR Pathway & $(9,11,12)$ \\
\hline TSC2 & Mut & 16p13.3 & 6 & PI3K/Akt/mTOR Pathway & $(9,11,12)$ \\
\hline TERT & Promoter mut & $5 p 15.33$ & 7 & Telomerase pathway & $(9,11,12)$ \\
\hline
\end{tabular}

The frequency was obtained using data from a combined study in The Cancer Genome Atlas (TCGA) database (https://www.cbioportal.org/). Skin Cutaneous Melanoma (TCGA, Firehose Legacy, N=479 samples), Skin Cutaneous Melanoma (TCGA, PanCancer Atlas), Skin Cutaneous Melanoma (Broad, Cell 2012) (9), Skin Cutaneous Melanoma (Yale, Nat Genet 2012) (11), and Skin Cutaneous Melanoma (Broad, Cancer Discov 2014) (12) were all included in the combined study. MUT, mutation; Amp, amplification; Del, deletion; Hm, hypermethylation; Freq, Frequency. 
of SMGs, cutaneous melanomas can be classified into four genomic subgroups, including mutant BRAF, mutant RAS, mutant NF1, and Triple-WT (wild-type) (8).

Braf is a component of the RAS/MAPK signaling pathway, which regulates a variety of critical cellular processes, such as cell proliferation, differentiation, migration, and apoptosis (Figure 1). BRAF somatic mutations were identified in $52.2 \%$ of 318 patients. V600E $(\mathrm{n}=124), \operatorname{V600K}(\mathrm{n}=18)$, and V600R $(\mathrm{n}=3)$ were among the 145 patients that targeted the V600 amino acid residue. The K601 residue was the target of the second most common BRAF mutation $(\mathrm{n}=5)$. BRAF V600 and K601 hot-spot mutations were negatively associated with hot-spot NRAS mutations. BRAF nonhot-spot mutations, on the other hand, frequently co-occurred with RAS hot-spot and NF1 mutations. The Nras protein, a GTPase, converts GTP to GDP. NRAS hot-spot mutations result in decreased GTPase activity, which leads to enhanced GTPbound Nras and activation of downstream pathways, such as RAF/MEK, PI3K/AKT, and RALGEF/RALA/RALB pathway (13, 14). NRAS somatic mutations were identified in $28.4 \%$ of 88 patients. Among them, 97.7\% $(n=88)$ of these patients harbored NRAS hot-spot mutations, including Q61R ( $\mathrm{n}=33), \mathrm{Q} 61 \mathrm{~K}(\mathrm{n}=28)$, Q61L ( $\mathrm{n}=11)$, Q61H $(\mathrm{n}=4), 61 \_62 \mathrm{QE}>\mathrm{HK}(\mathrm{n}=1)$, and G13R/D $(n=3)$. Other RAS family members, such as hot-spot HRAS (G13D, G13S, and Q61K) and KRAS (G12D, G12R, and Q61R), were shown to have mutations that were mutually exclusive with NRAS and BRAF V600 and K601 mutations. Patients carrying BRAF mutations were considerably younger than those carrying

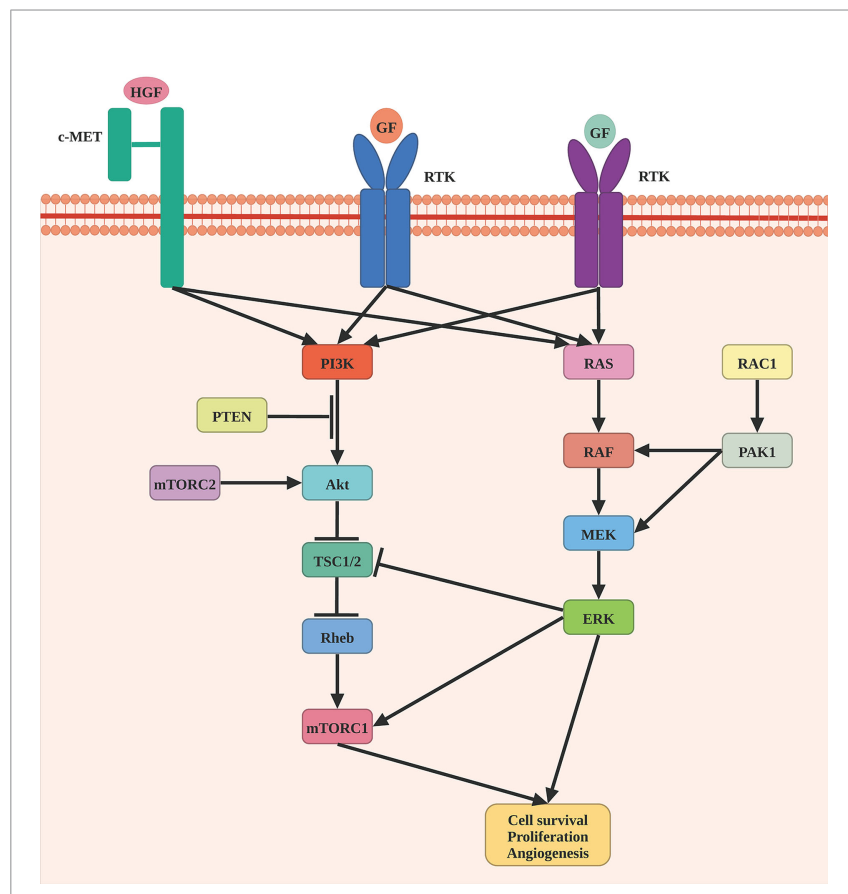

FIGURE 1 | Schematic representation of MAPK pathway in melanomagenesis. Oncogenic mutations in BRAF are the most common genomic alterations in melanoma, followed by a mutation in NRAF, NF1, and RAC1. other mutations, while those with NF1 mutations tended to be older. NF1 mutation was detected in $14 \%$ of cases, most of which are loss-of-function events. Nf1 is a Ras pathway negative regulator that may stimulate RAS GTPase activity, causing cell growth and malignancy. Besides, the MAPK signaling pathway can be stimulated by loss-of-function mutation of NF1. Moreover, the NF1 subtype exhibited a significantly high mutation burden $(8,15,16)$.

BRAF, MITF, and CD274 amplifications were found at substantial frequencies in patients with BRAF mutations, while NRAS amplifications occurred in patients with NRAS mutations. PTEN deletions and mutations were more prevalent in the BRAF-mutant subgroup, while mRNA overexpression and amplification of AKT3 were considerably more prevalent in RAS, NF1, and Triple-WT subtypes, which may serve as new biomarkers to justify the use of MEK and PI3K/AKT/mTOR pathway inhibitors in these subtypes. Moreover, a UV signature was observed in $90.7 \%(136 / 151)$ of BRAF, $93.5 \%$ (86/92) of RAS, and $92.9 \%(26 / 28)$ of NF1 subtypes but only $30 \%(14 / 46)$ of Triple-WT. Although the Triple-WT lacked a UV signature, this subtype exhibited high copy-number alterations and complex rearrangements. KIT, PDGFRA, KDR, CDK4 and CCND1, MDM2, and TERT amplifications were frequently observed in Triple-WT melanomas. Besides, TERT promoter mutations were identified in $75.0 \%(n=52)$ of BRAF, $71.9 \%(n=32)$ of RAS, and $83.3 \%(n=12)$ of NF1 subtypes but in only $6.7 \%(n=15)$ of TripleWT (8).

CDKN2A is notable for encoding two vastly distinct proteins: p16INK4a and p14ARF. p16INK4a functions as a cyclindependent kinase inhibitor in the RB1 cell cycle pathway, while p14ARF interacts with the p53-stabilizing protein MDM2 in the p53 pathway. Alterations in CDKN2A in melanoma mainly target p16INK4a or affect both p16INK4a and p14ARF. Additionally, there is a subgroup of less frequent somatic and germline INK4a/ARF alterations that impact p14ARF but not p16INK4a (17). Bi-allelic loss of CDKN2A is correlated with aggressive initiation of melanoma. BRN2 is transcriptionally triggered by the loss of p16INK4A through direct binding to E2F1 due to CDK4/6-mediated hyperphosphorylation of RB1 (18).

RAC1 encodes a GTPase that is a member of the Ras superfamily of small GTP-binding proteins. Numerous protein kinases act as RAC1 effectors, providing an avenue for pharmacological suppression. The RAC1 P29S mutation is a frequent UV-signature mutation that is found in $9.2 \%$ of sunexposed melanomas. The RAC1 P29S mutation was identified in both primary $(9.2 \%)$ and metastatic $(8.6 \%)$ melanoma, indicating that it is present early in carcinogenesis (11). In melanocytes, RAC1P29S stimulates PAK, AKT, and the SRF/MRTF transcription pathway and promotes a shift from melanocytic to mesenchymal cells through SRF/MRTF and PAK. Besides, RAC1 P29S in conjunction with BRAF mutations or NF1 deletions stimulates tumorigenesis. RAC1 P29S confers resistance to BRAF inhibitors through the SRF/MRTF signaling pathway, indicating that targeted SRF/MRTF therapy may overcome BRAF inhibitor resistance in melanoma patients 
with RAC1P29S mutations. Moreover, RAC1 P29S exhibited enhanced binding to PAK1, MLK3, and the WAVE complex. MLK3 can recruit a BRAF-RAF1 complex, implying that it may serve as a link between the RAC1 and MAP kinase cascades. Therebefore, RAC1 may be a therapeutic target in melanoma $(11,19)$.

\section{THERAPY TARGETING BRAF AND MEK}

In 2011, the Food and Drug Administration (FDA) approved vemurafenib as the first BRAF targeted therapy for unresectable or metastatic BRAF V600E-mutated melanoma based on an overall survival rate of $84 \%$ and a $63 \%$ decrease in the risk of mortality from the BRIM 3 trial (20). Despite its clinical significance, vemurafenib monotherapy rapidly develops resistance via reactivating mitogen-activated protein (MAP) kinase. The combination of BRAF and MEK inhibition that tackle this resistance mechanism has become the standard of treatment for melanoma patients. Targeted therapy with BRAF inhibitor in conjunction with MEK inhibitor has long-term benefits, but acquired resistance may restrict long-term disease management (Table 2 and Figure 2).

Based on the COMBI-v trial (dabrafenib plus trametinib vs. vemurafenib) (21) and COMBI-d trial (dabrafenib plus trametinib to dabrafenib) (22), the 5-year progression-free survival rates and overall rates for unresectable or metastatic melanoma with a BRAF V600 mutation treated with dabrafenib plus trametinib as first-line therapy were 19\% and 34\%, respectively (23). The coBRIM trial exhibited that first-line vemurafenib combined with cobimetinib improved progression-free and overall survival compared with vemurafenib in patients with BRAF V600-mutated advanced melanoma. The median overall survival of cobimetinib and

TABLE 2 | Melanoma treatment options (targeted and immune therapies).

\begin{tabular}{|c|c|c|c|}
\hline Drugs & Drug targets & Mechanism & Indications \\
\hline Ipilimumab & CTLA-4 & $\begin{array}{l}\text { Anti-CTLA-4 } \\
\text { inhibitor }\end{array}$ & Unresectable or metastatic melanoma (NCT00094653; 2011) \\
\hline Vemurafenib & $\begin{array}{l}\text { BRAF V600E, CRAF, ARAF, wild-type } \\
\text { BRAF, SRMS, ACK1, MAP4K5 and } \\
\text { FGR }\end{array}$ & BRAF inhibitor & $\begin{array}{l}\text { Unresectable or metastatic melanoma with the BRAF V600E mutation (NCT01006980; } \\
\text { 2011) }\end{array}$ \\
\hline Trametinib & MEK1, MEK2 & MEK inhibitor & $\begin{array}{l}\text { Unresectable or metastatic melanoma with BRAF V600E or V600K mutation } \\
\text { (NCT01245062; 2013) }\end{array}$ \\
\hline Dabrafenib & $\begin{array}{l}\text { BRAF V600, wild-type BRAF, CRAF } \\
\text { kinases, SIK1, NEK11, and LIMK1 }\end{array}$ & BRAF inhibitor & $\begin{array}{l}\text { Unresectable or metastatic melanoma with BRAF V600E mutation (NCT01227889; } \\
\text { 2013) }\end{array}$ \\
\hline $\begin{array}{l}\text { Dabrafenib } \\
\text { +trametinib }\end{array}$ & $\begin{array}{l}\text { BRAF V600, wild-type BRAF, CRAF } \\
\text { kinases, SIK1, NEK11, and LIMK1; } \\
\text { MEK1, MEK2 }\end{array}$ & $\begin{array}{l}\text { BRAF inhibitor } \\
\text { +MEK inhibitor }\end{array}$ & $\begin{array}{l}\text { Unresectable or metastatic melanoma with a BRAF V600E or V600K mutation } \\
\text { (NCT01584648; 2014) }\end{array}$ \\
\hline Pembrolizumab & PD-1 & Anti-PD-1 inhibitor & $\begin{array}{l}\text { Unresectable or metastatic melanoma with disease progression following treatment with } \\
\text { ipilimumab and, in BRAF V600 mutation-positive patients after treatment with a BRAF } \\
\text { inhibitor (NCT01295827; 2014) }\end{array}$ \\
\hline Nivolumab & PD-1 & Anti-PD-1 inhibitor & $\begin{array}{l}\text { Unresectable or metastatic melanoma with disease progression following treatment with } \\
\text { ipilimumab and, in BRAF V600 mutation-positive patients after treatment with a BRAF } \\
\text { inhibitor (NCT01721772; 2014) }\end{array}$ \\
\hline $\begin{array}{l}\text { Vemurafenib } \\
\text { +cobimetinib }\end{array}$ & $\begin{array}{l}\text { BRAF V600E, CRAF, ARAF, wild-type } \\
\text { BRAF, SRMS, ACK1, MAP4K5 and } \\
\text { FGR; MEK1, MEK2 }\end{array}$ & $\begin{array}{l}\text { BRAF inhibitor } \\
\text { +MEK inhibitor }\end{array}$ & $\begin{array}{l}\text { Unresectable or metastatic melanoma with BRAF V600E or V600K mutation } \\
\text { (NCT01689519; 2015) }\end{array}$ \\
\hline $\begin{array}{l}\text { Nivolumab } \\
+ \text { ipilimumab }\end{array}$ & PD-1; CTLA-4 & $\begin{array}{l}\text { Anti-PD-1 inhibitor } \\
+ \text { Anti-CTLA-4 } \\
\text { inhibitor }\end{array}$ & BRAF V600 wild-type, unresectable or metastatic melanoma (NCT01844505; 2015) \\
\hline Ipilimumab & CTLA-4 & $\begin{array}{l}\text { Anti-CTLA-4 } \\
\text { inhibitor }\end{array}$ & $\begin{array}{l}\text { Adjuvant treatment of patients with cutaneous melanoma with pathologic involvement of } \\
\text { regional lymph nodes of more than } 1 \mathrm{~mm} \text { who have undergone complete resection, } \\
\text { including total lymphadenectomy (NCT00636168; 2015) }\end{array}$ \\
\hline Nivolumab & PD-1 & Anti-PD-1 inhibitor & $\begin{array}{l}\text { Adjuvant treatment of patients with melanoma with involvement of lymph nodes or in } \\
\text { patients with metastatic disease who have undergone complete resection } \\
\text { (NCT02388906; 2017) }\end{array}$ \\
\hline $\begin{array}{l}\text { Encorafenib } \\
\text { +binimetinib }\end{array}$ & $\begin{array}{l}\text { BRAF V600, JNK1, JNK2, JNK3, } \\
\text { LIMK1, LIMK2, MEK4, and STK36; } \\
\text { MEK1, MEK2 }\end{array}$ & $\begin{array}{l}\text { BRAF inhibitor } \\
\text { +MEK inhibitor }\end{array}$ & $\begin{array}{l}\text { Unresectable or metastatic melanoma with a BRAF V600E or V600K mutation } \\
\text { (NCT01909453; 2018) }\end{array}$ \\
\hline $\begin{array}{l}\text { Dabrafenib } \\
\text { +trametinib }\end{array}$ & $\begin{array}{l}\text { BRAF V600, wild-type BRAF, CRAF } \\
\text { kinases, SIK1, NEK11, and LIMK1; } \\
\text { MEK1, MEK2 }\end{array}$ & $\begin{array}{l}\text { BRAF inhibitor } \\
\text { +MEK inhibitor }\end{array}$ & $\begin{array}{l}\text { Adjuvant treatment of patients with melanoma with BRAF V600E or V600K mutations } \\
\text { (NCT01682083; 2018) }\end{array}$ \\
\hline Pembrolizumab & PD-1 & Anti-PD-1 inhibitor & $\begin{array}{l}\text { Adjuvant treatment of patients with melanoma with involvement of lymph node(s) } \\
\text { following complete resection (NCT02362594; 2019) }\end{array}$ \\
\hline $\begin{array}{l}\text { Atezolizumab } \\
\text { + vemurafenib } \\
\text { +cobimetinib }\end{array}$ & $\begin{array}{l}\text { PD-L1; BRAF V600E, CRAF, ARAF, } \\
\text { wild-type BRAF, SRMS, ACK1, } \\
\text { MAP4K5 and FGR; MEK1, MEK2 }\end{array}$ & $\begin{array}{l}\text { Anti-PD-L1 } \\
\text { inhibitor+BRAF } \\
\text { inhibitor+MEK } \\
\text { inhibitor }\end{array}$ & $\begin{array}{l}\text { BRAF V600 mutation-positive unresectable or metastatic melanoma (NCT02908672; } \\
\text { 2020) }\end{array}$ \\
\hline
\end{tabular}






FIGURE 2 | Timeline of FDA-approved melanoma therapies. T-VEC, talimogene laherparepvec.

vemurafenib (22.5 months) was superior to that of vemurafenib (17.4 months) after a median follow-up of 21.2 months. In addition, cobimetinib combined with vemurafenib had a median progression-free survival of 12.6 months versus 7.2 months for vemurafenib (24-26). In the COLUMBUS trial, the median overall survival of patients with BRAF V600-mutant melanoma was 33.6 months with COMBO450 (encorafenib plus binimetinib), 23.5 months with ENCO300 (encorafenib), and 16.9 months with VEM (vemurafenib). COMBO450 reduced the risk of mortality by $39 \%$ when compared to VEM. The median progression-free survival was 14.9 months with COMBO450, 9.6 months with ENCO300, and 7.3 months with VEM $(27,28)$. Overall, treatment with combined BRAF and MEK inhibition, such as dabrafenib plus trametinib, vemurafenib plus cobimetinib, and encorafenib plus binimetinib seems to provide longer progression-free and overall survival compared to treatment with BRAF inhibitor monotherapy.

The five-year analysis of adjuvant dabrafenib with trametinib in stage III melanoma with BRAF V600 mutations was presented in the COMBI-AD trial. With dabrafenib plus trametinib, $52 \%$ of patients survived without recurrence, compared to $36 \%$ with placebo. Moreover, $65 \%$ of patients survived without distant metastasis with dabrafenib plus trametinib, compared to $54 \%$ with placebo $(29,30)$. The NeoCombi study showed that among resectable, stage III, BARF V600 mutation melanoma, neoadjuvant dabrafenib plus trametinib therapy contributed to a RECIST response in $86 \%$ of patients and a complete pathological response with no progression in $49 \%$ of patients during neoadjuvant therapy (31).

Several hotspots of the KIT gene are oncogenic, and these hotspots exhibit variable responsiveness to KIT inhibitors, such as imatinib, sunitinib, nilotinib. Patients with metastatic melanoma harboring KIT mutation or amplification who received imatinib had a median progression-free survival of 3.5 months and a 6month PFS rate of $36.6 \%$ (32). The appearance of mutations in KIT exons 11 and 13, such as W557, V559, L576P, K642E exhibited a high degree of sensitivity to KIT inhibition. KIT exon 17 mutations such as D816H seem to be less sensitive to KIT inhibitors. KIT amplification or NRAS mutations seem to be less sensitive or insensitive to KIT inhibitors (33-35).

The NEMO trial exhibited that advanced NRAS-mutant melanoma patients receiving binimetinib had a median progression-free survival of 28 months while advanced NRASmutant melanoma patients receiving dacarbazine had a median progression-free survival of 15 months after a median follow-up of 1.7 months (36).

\section{POTENTIAL MECHANISMS OF RESISTANCE TO TARGETED THERAPY}

Most melanomas had oncogenic alterations such as RAC1 and AKT3 that stimulate the MAPK and PI3K pathways, reducing sensitivity to MAPK inhibitors (37). SEC61-dependent ER translocation of the MAPK pathway was triggered by BRAF and MEK inhibitors through GRP78 and KSR2. ER translocation inhibition inhibited ERK reactivation and autophagy. ERK reactivation contributed to the phosphorylation of AFT4, which triggered cytoprotective autophagy. In patients resistant to BRAF and MEK inhibitors, GRP78 upregulation and ATF4 phosphorylation were found, suggesting that ER translocation of the MAPK pathway causes treatment resistance in BRAF-mutant melanoma (38). PAKs phosphorylated CRAF and MEK to stimulate ERK in cells that are resistant to BRAF inhibitor. In cells resistant to combined BRAF and MEK inhibition, PAKs 
modulate the phosphorylation of JNK and $\beta$-catenin and the activation of the mTOR pathway, supporting that PAK signaling is responsible for acquired resistance to MAPK inhibitors in BRAF-mutant melanoma (39). Belvarafenib is a selective RAF dimer (type II) inhibitor with clinical efficacy in BRAF and NRAS-mutant melanomas. ARAF mutants develop resistance to belvarafenib in a kinase- and dimer-dependent manner. Combined RAF and MEK inhibition may postpone ARAFdriven resistance (40). The RAC1 P29S mutation works along with BRAF to promote melanoma initiation and cause resistance to BRAF inhibitors. In melanoma, the transcription factor complex SRF/MRTF is a key RAC1 effector, which has generated a therapeutic resistance by inducing a mesenchymallike state (19). SPRED1 inactivation is common in BRAFmutated melanoma. SPRED1 deficiency enhanced melanoma cell proliferation under mutant BRAF inhibition via the reactivation of MAPK activity. SPRED1 loss has been found in patients whose melanoma had developed resistance to MAPKtargeted treatment (41). Combined MEK and CDK4/6 inhibition is a promising therapeutic option in mutant BRAF and NRAS melanoma. The ribosomal S6 protein phosphorylation promotes the activation of the mTOR signaling pathway and leads to acquired drug resistance, supporting that targeting mTORC1/2 may overcome MEK plus CDK4/6 inhibition resistance (42). In patients with NRAS-mutant melanoma, combined MEK and CDK4/6 inhibitors have demonstrated encouraging therapeutic results. However, an acquired PIK3CA E545K mutation can depend on the S6K1 signaling pathway for drug resistance. S6K1 inhibition may resensitize NRAS-mutant melanoma carrying PIK3CA E545K to combined MEK and CDK4/6 inhibition (43).

Due to primary and secondary resistance, MEK inhibitors have shown limited effectiveness in patients with NRAS-mutated melanoma. Two patients with NRAS-mutant metastatic melanoma had a long-term response to intermittent binimetinib (MEK inhibitor). Intermittent administration may help overcome resistance, which is associated with the fact that it may lead to a fitness deficit for drug-resistant cells, enhance immunogenicity, enhance expression of immunomodulatory molecules, reduce immunosuppressive factors, and induce apoptosis and cell cycle arrest (44).

Exploratory biomarker analyses from the COMBI-AD study (NCT01682083) exhibited that the presence of MAP2K1/MEK1 mutations, BRAF amplification, or MAPK pathway gene (NRAS, KRAS, HRAS, MAP2K1/MEK1, MAP2K2/MEK2, MAPK1, NF1, MAPK7, or MAPK3) mutations at baseline did not affect clinical benefit. A significant increase in relapse-free survival was observed in patients with a high IFN $\gamma$ gene expression signature in the dabrafenib plus trametinib group and placebo group. Tumor mutational burden (TMB) was independently associated with a higher likelihood of relapse-free survival in the placebo group. However, TMB was not associated with relapse-free survival in patients treated with dabrafenib plus trametinib. Besides, patients with a lower TMB benefited more from dabrafenib plus trametinib adjuvant treatment. Specifically, the subgroup with low TMB and high IFN gene expression signature benefited the most from targeted treatment (45).
Baseline genetic characteristics of BRAF V600-mutated metastatic melanoma patients treated with cobimetinib plus vemurafenib or vemurafenib alone showed that MITF and TP53 alterations were more frequent in patients who progressed rapidly, while NF1 alterations were more frequent in patients who obtained a complete response, which is consistent with the view that melanomas that lack NF1 expression are more dependent on the MAPK signaling pathway and are more sensitive to MAPK pathway inhibitors. Additionally, immune-related gene signatures such as CD8+ effector T cells, cytolytic T cells, antigen-presenting cells, and natural killer cells gene signatures were associated with complete response, while keratinization-related gene expression was associated with rapid progression (46).

\section{IMMUNE CHECKPOINT INHIBITORS}

Metastatic melanoma can benefit from immunotherapy, which can regulate and activate the immune system against cancer. The presence of lymphocytic infiltration is a hallmark of primary melanoma. The cytotoxic antigen 4 (CTLA-4) and the programmed death 1 (PD-1) immune checkpoints are the negative $\mathrm{T}$-cell immune function modulators (Table 2). Inhibiting CTLA-4 and PD-1/PD-L1, leading to enhanced immune system activation, has contributed to the development of novel melanoma immunotherapies. During the interaction between antigen-presenting cells and T cells, CTLA-4, which functioned as a negative $\mathrm{T}$-cell activation modulator in lymphoid tissues, is up-regulated on $\mathrm{T}$ cells due to the binding of tumor antigens to T-cell receptors. Modulating PD-L1 expression can reduce immune monitoring in the tumor microenvironment and prevent tumor immune escape by inhibiting $\mathrm{T}$ cell activation, implying that PD-L1/PD-1 signaling pathway is one of the main pathways of tumor immune escape. Approximately $30 \%$ of patients with metastatic melanoma who received anti-PD-1 therapy achieve long-term disease control. However, about two-thirds of individuals suffer from resistance and require further treatment (Figure 3).

A pooled analysis including KEYNOTE-001, KEYNOTE-002, and KEYNOTE-006 study endorses the use of pembrolizumab for treatment of advanced melanoma independent of BRAF V600 mutation status or previous treatment with BRAF inhibitor with or without MEK inhibitor. For patients with BRAF wild-type and BRAF V600-mutant melanoma, the objective response rate was $39.8 \%(447 / 1124)$ and $34.3 \%$ (149/434), 4-year progression-free survival rate was $22.9 \%(257 / 1124)$ and $19.8 \%(86 / 434)$, and 4 -year overall survival was 37.5\% (421/1124) and 35.1\% (152/434), respectively. For patients with BRAF V600-mutant melanoma previously treated with or without BRAF inhibitor in combination with or without MEK inhibitor, the objective response rate was $28.4 \%(77 / 271)$ and $44.2 \%(72 / 163)$, 4-year progression-free survival rate was $15.2 \%(41 / 271)$ and $27.8 \%$ $(45 / 163)$, and 4-year overall survival was $26.9 \%(73 / 271)$ and $49.3 \%$ (80/163), respectively (47). In the KEYNOTE-002 (NCT01704287) trial, pembrolizumab had an improvement in 


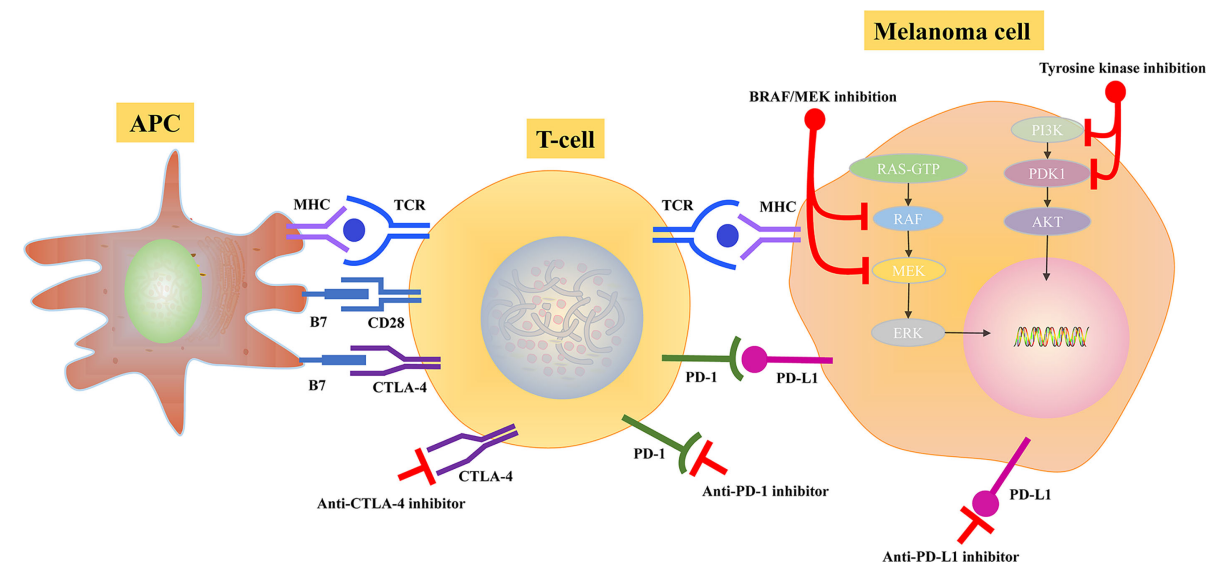

FIGURE 3 | Potential mechanisms of BRAF/MEK inhibitor and immune checkpoint inhibition in melanoma.

OS in patients with advanced melanoma compared with chemotherapy, but this was not statistically significant. The median OS was 13.4 months in the pembrolizumab $2 \mathrm{mg} / \mathrm{kg}$ arm, 14.7 months in the pembrolizumab $10 \mathrm{mg} / \mathrm{kg}$ arm, and 11.0 months in the chemotherapy arm, respectively. The two-year survival rates for the pembrolizumab $2 \mathrm{mg} / \mathrm{kg}$, pembrolizumab 10 $\mathrm{mg} / \mathrm{kg}$, and chemotherapy groups were $36 \%, 38 \%$, and $30 \%$, respectively. Grade 3 or 4 treatment-related AEs occurred in $13.5 \%(\mathrm{~N}=178)$ of the patients who received pembrolizumab 2 $\mathrm{mg} / \mathrm{kg}, 16.8 \%(\mathrm{~N}=179)$ of the patients who received pembrolizumab $10 \mathrm{mg} / \mathrm{kg}$, and $26.3 \%(\mathrm{~N}=171)$ of the patients who received chemotherapy (48). In the KEYNOTE-006 study, the pembrolizumab groups had a median overall survival of 32.7 months, whereas the ipilimumab group had a median overall survival of 15.9 months after a median follow-up of 57.7 months. In addition, the pembrolizumab groups had a median progression-free survival of 8.4 months compared to 3.4 months in the ipilimumab group. Grade 3 or 4 treatment-related AEs occurred in $17 \%(\mathrm{~N}=555)$ of patients who received pembrolizumab, and $20 \%(\mathrm{~N}=256)$ of patients who received ipilimumab. Colitis (2\% in the pembrolizumab groups and $6 \%$ in the ipilimumab group), diarrhoea ( $2 \%$ in the pembrolizumab groups and $3 \%$ in the ipilimumab group), and fatigue $(<1 \%$ in the pembrolizumab groups and $1 \%$ in the ipilimumab group) were the most common grade 3 or 4 treatment-related AEs. $14 \%(\mathrm{~N}=536)$ of patients in the pembrolizumab arm and $18 \%(\mathrm{~N}=250)$ of patients in the ipilimumab group experienced any grade serious treatment-related AEs. In the pembrolizumab arm, one patient died from treatment-related sepsis. After nearly 5 years of followup, pembrolizumab demonstrated superiority over ipilimumab, supporting its usage in patients with advanced melanoma (49).

The CheckMate 037 study (NCT01721746) revealed that advanced melanoma patients who received nivolumab had more and higher durable responses than those who received chemotherapy. Median OS was 16 months versus 14 months. Median progression-free survival was 3.1 months in the nivolumab group and 3.7 months in the chemotherapy group.
The overall response rate was $27 \%$ and $10 \%$ in the nivolumab and chemotherapy groups, respectively. The median response duration was 32 months in the nivolumab arm and 13 months in the chemotherapy group. Fewer treatment-related adverse events (AEs) (incidence $\geq 5 \%$ ) were found in patients on nivolumab (77\% vs. $82 \%$ ). Grade 3 or 4 AEs occurred in $14 \%$ and $34 \%$ of patients in the nivolumab and chemotherapy groups, respectively. The most common AEs (incidence $\geq 10 \%$ ) in patients receiving nivolumab were fatigue, pruritus, diarrhea, rash, nausea, and vitiligo (50). In the CheckMate 066 trial, the 5year overall survival rate was $39 \%$ with nivolumab, $17 \%$ with dacarbazine, and $38 \%$ with dacarbazine and subsequent therapy, including nivolumab in patients with wild-type BRAF advanced melanoma. The 5-year analysis confirmed that nivolumab had a significant benefit over dacarbazine and extended long-term survival (51).

After a median follow-up of 22.1 months, the objective response rate of ipilimumab plus anti-PD-1 (pembrolizumab or nivolumab) and ipilimumab monotherapy was 31\% (60/193) and 13\% (21/162) for patients with advanced melanoma who had previously failed anti-PD-1 or anti-PD-L1 monotherapy. The overall survival of ipilimumab plus anti-PD-1 and ipilimumab monotherapy was 20.4 months and 8.8 months $(\mathrm{p}<0.0001)$, respectively. Besides, the progression-free survival was longer in the ipilimumab plus antiPD-1 group than ipilimumab group ( 3.0 vs. 2.6 months; $\mathrm{p}=0.0019$ ). Grade 3-5 AEs occurred in 31\% ( $\mathrm{N}=193)$ of the patients in the ipilimumab plus anti-PD-1 inhibitor group compared with 33\% $(\mathrm{N}=162)$ in the ipilimumab alone group. The most common 3-5 AEs in the ipilimumab plus anti-PD-1 inhibitor group were diarrhoea or colitis (12\%), and increased alanine aminotransferase or aspartate aminotransferase (12\%). In the ipilimumab alone group, the most common 3-5 AEs were diarrhoea or colitis $(20 \%)$, and increased alanine aminotransferase or aspartate aminotransferase (9\%). The result indicated that ipilimumab combined with anti-PD-1 was superior to ipilimumab monotherapy as second-line immunotherapy for patients with advanced melanoma (52). The CheckMate 067 study exhibited 
that the median overall survival was dramatically improved with nivolumab plus ipilimumab compared to nivolumab or ipilimumab (over 60.0 vs. 36.9 vs. 19.9 months) after a minimum follow-up of 5 years in advanced melanoma. Besides, advanced melanoma patients treated with nivolumab plus ipilimumab had a 5-year overall survival rate of $52 \%$, compared with $44 \%$ for nivolumab and only $26 \%$ for ipilimumab. The 5-year overall survival rates of patients with BRAF mutation treated with nivolumab plus ipilimumab, nivolumab, and ipilimumab were $60 \%, 46 \%$, and $30 \%$, respectively. The 5-year overall survival rates of patients without BRAF mutation treated with nivolumab plus ipilimumab, nivolumab, and ipilimumab were $48 \%, 43 \%$, and $25 \%$, respectively. Grade 3 or 4 treatment-related AEs occurred in 59\% of patients who received nivolumab plus ipilimumab, $23 \%$ of patients who received nivolumab, and $28 \%$ of patients who received ipilimumab. Neither nivolumab alone nor nivolumab plus ipilimumab exhibited a significant decline in health-related quality of life. The findings showed that nivolumab plus ipilimumab or nivolumab alone might help patients with advanced melanoma achieve longer overall survival than ipilimumab (53). The response rates of anti-PD-1 inhibitors to patients with melanoma was 30$40 \%$. When immune checkpoint therapy is combined with anti-PD1 and anti-CTLA- 4 inhibitors, the rate of innate resistance is reduced from $60-70 \%$ to $40-50 \%$. Anti-PD-1/L1 before MAPK inhibitor treatment extends the duration of tumor remission. Prior immune checkpoint therapies are linked to prolonged progression-free survival in melanoma patients treated with MAPK inhibitor. Targeting M2-like tumor-associated macrophages (TAMs) and enhancing the clonal expansion of tumor-specific CD8+T cells may enhance anti-PD-L1 efficacy. In addition, prioritizing treatment with anti-PD1/L1 plus anti-CTLA-4 before MAPK inhibitor combination limits melanoma brain metastases (54).

In the COMBI-i trial, the objective response rate of combined spartalizumab (anti-PD-1 inhibitor), dabrafenib, and trametinib was $78 \%$ in advanced BRAF-mutant melanoma, and $44 \%$ of patients achieved a complete response. All patients experienced treatment-related AEs, with $72 \%$ suffering grade 3 or higher treatment-related AEs. The most common $(\geq 8 \%)$ grade 3 or higher AEs were pyrexia, increased lipase, increased gammaglutamyltransferase, and neutropenia increased blood creatine phosphokinase. $17 \%(\mathrm{~N}=35)$ of patients were discontinued due to treatment-related AEs, including immune-mediated hepatitis, paresthesia, hypokalemia, interstitial lung disease, increased alanine and aspartate aminotransferases, increased gammaglutamyltransferase and generalized exfoliative dermatitis (55). The IMspire150 study exhibited that after a median follow-up of 18.9 months, progression-free survival was substantially longer in the atezolizumab group (vemurafenib, cobimetinib, and atezolizumab) than in the placebo group (vemurafenib, cobimetinib, and atezolizumab placebo) (15.1 vs. 10.6 months). The most common treatment-related AEs (incidence $>30 \%$ ) in patients receiving atezolizumab plus vemurafenib and cobimetinib are blood creatinine phosphokinase increased (51.3\%), diarrhoea (42.2\%), rash (40.9\%), arthralgia $(39.1 \%)$, pyrexia $(38.7 \%)$, alanine aminotransferase increased $(33.9 \%)$, and lipase increased $(32 \cdot 2 \%)$. The most common treatmentrelated AEs (incidence $>30 \%$ ) to atezolizumab placebo plus vemurafenib and cobimetinib are blood creatinine phosphokinase increased $(44.8 \%)$, diarrhoea $(46.6 \%)$, rash $(40.9 \%)$, arthralgia $(28 \cdot 1 \%)$, pyrexia $(26 \cdot 0 \%)$, alanine aminotransferase increased $(22.8 \%)$, and lipase increased $(27 \cdot 4 \%)$. In the atezolizumab group, $13 \%$ of patients and in the control group, $16 \%$ of patients discontinued therapy due to AEs. The study (IMspire150) revealed that atezolizumab was safe and tolerable when combined with vemurafenib and cobimetinib, and substantially improved progression-free survival in patients with BRAF V600 mutation-positive advanced melanoma (56).

The PIVOT-02 study (NCT02983045) exhibited the safety and efficacy of bempegaldesleukin, a CD122-preferential interleukin-2 pathway agonist, plus nivolumab in the first-line treatment of metastatic melanoma. After a median follow-up of 29.0 months, the objective response rate was $52.6 \%(\mathrm{~N}=38)$, and the complete response rate was $34.2 \%(\mathrm{~N}=38)$. Besides, the median progression-free survival of metastatic melanoma was 30.9 months. Bempegaldesleukin plus nivolumab caused grade 3 or 4 treatment-related AEs in $17.1 \%(\mathrm{~N}=41)$ of patients, which is consistent with anti-PD-1 inhibitors (16-17\%) in this setting and substantially lower than nivolumab plus ipilimumab (55\%) and BRAF plus MEK inhibitors (54\%-68\%) (57).

The EORTC 1325-MG/KEYNOTE-054 trial endorsed the use of adjuvant pembrolizumab treatment in patients with high-risk stage III melanoma. The adjuvant pembrolizumab group had a better 3.5-year distant metastasis-free survival rate after a median follow-up of 42.3 months compared with the placebo group in the intention-to-treat population (65.3\% vs. 49.4\%) (58). Besides, the adjuvant pembrolizumab group had a longer recurrence-free survival compared with the placebo group in the intention-totreat population (Hazard ratio [HR], 0.56 ; $98.4 \% \mathrm{CI}, 0.43$ to 0.74 ). In addition, the occurrence of immune-related adverse events was linked to prolonged recurrence-free survival in the pembrolizumab group (59). In the KEYNOTE-716 (NCT03553836) trial, patients with resected high-risk stage II melanoma who received pembrolizumab in the adjuvant setting had a $35 \%$ reduction in disease recurrence or mortality compared to placebo (60). Patients with high-risk stage III melanoma treated with pembrolizumab had a 3-year recurrence-free survival rate of $63.7 \%$, compared with $44.1 \%$ for placebo (61). For patients with resected high-risk melanoma, adjuvant ipilimumab therapy $(3 \mathrm{mg} / \mathrm{kg})$ had a significant difference in overall survival compared with high-dose interferon alfa-2b therapy $(\mathrm{HR}, 0.78 ; 95.6 \% \mathrm{CI}, 0.61$ to $0.99 ; \mathrm{P}=$ 0.044) in the E1609 trial (62). In the CheckMate 238 trial, adjuvant nivolumab showed a prolonged recurrence-free survival benefit compared with ipilimumab in resected stage IIIB-C or IV melanoma at a minimum follow-up of 4 years $(51.7 \%$ vs. $41.2 \%$, $\mathrm{P}=0.0003$ ) (63). The IMMUNED trial compared the effectiveness of adjuvant nivolumab plus ipilimumab or nivolumab monotherapy to placebo in patients with resected stage IV melanoma. The median recurrence-free survival was not reached in the nivolumab plus ipilimumab arm, but the median recurrencefree survival in the nivolumab arm was 12.4 months and 6.4 months in the placebo arm after a median follow-up of 28.4 
months (64). The OpACIN and OpACIN-neo trials exhibited that neoadjuvant ipilimumab plus nivolumab had high pathologic response rates in patients with macroscopic stage III melanoma. None of the patients in the OpACIN study with a pathologic response (7/9) had relapsed after a median follow-up of 4 years. Additionally, 2-year relapse-free survival was $84 \%$ for all patients, $97 \%$ for those who achieved a pathological response, and $36 \%$ for those who did not in the Opacin-neo study (65). In a pooled analysis, neoadjuvant therapy in melanoma with ipilimumab plus nivolumab, anti-PD-1 inhibitor, and targeted inhibitor showed pathological complete response rates of $43 \%, 20 \%, 47 \%$, respectively (66).

\section{ADOPTIVE CELL TRANSFER (ACT)}

Adoptive cell transfer (ACT) utilizing tumor-infiltrating lymphocytes or modified $\mathrm{T}$ cells has shown encouraging outcomes in patients with melanoma (67). Patients with metastatic melanoma who have progressed after receiving immune checkpoint inhibitors or targeted therapies have limited therapeutic alternatives. Tumor-infiltrating lymphocyteadministered adoptive cell therapy has shown to be effective in treating metastatic melanoma. Lifileuel is an autologous tumorinfiltrating lymphocyte (TIL) product that has shown sustained responses in patients with metastatic melanoma who have progressed following immune checkpoint inhibitors or targeted therapies. The ORR to lifileuel was 36\% $(\mathrm{N}=66)$ for metastatic melanoma, consisting of two complete responses and 22 partial responses, and the disease control rate reached $80 \%$ (68). In a clinical phase I/II study (NCT03296137), tumor-infiltrating lymphocytes (TILs)-based adoptive cell therapy (ACT) with immune checkpoint inhibitors was assessed across various solid cancer types. Five patients, including two partial responses in patients with cholangiocarcinoma and head-and-neck cancer, experienced significant tumor regressions of 30\%-63\%. Moreover, clinical effectiveness is related to the phenotypic characterization of rapid expansion protocol (REP) TILs, particularly alpha-integrin CD103 expression and tumor mutational burden (TMB). Additionally, the potential of employing immune checkpoint inhibitors to facilitate TIL development and treatment also needs to be investigated (69). The study of chimeric antigen receptor (CAR) T-cell treatments is progressing, but the anticipated benefits in hematologic malignancies have yet to be shown in solid tumors. The paucity of CAR-T cells that migrate from blood vessels to the target site, the immunosuppressive tumor microenvironment inside the malignant tumor, and the appropriate identification of target antigen to prevent on-target/off-tumor toxicities are all significant obstacles (70).

\section{CYTOKINES (INTERLEUKIN-2)}

Interleukin-2 (IL-2) is an important cytokine with a variety of effects on the immune system. It has extensive effects on the development and expansion of T cell subsets, particularly CD8+
$\mathrm{T}$ cells, and has anti-tumor activity in advanced melanoma. In 1998, the Food and Drug Administration (FDA) approved highdose IL-2 for the treatment of patients with metastatic melanoma. In a phase II trial in $1994,7 \%(\mathrm{~N}=129)$ of patients with metastatic melanoma experienced complete regression of disease and $10 \%(\mathrm{~N}=140)$ of patients experienced partial regression (71). In a retrospective study, the overall objective response rate in patients with metastatic melanoma treated with IL-2 was $16 \%(\mathrm{~N}=270)$, with 17 complete responses and 26 partial responses (72). In another retrospective study, high-dose IL-2 treatment maintained antitumor efficacy in patients with metastatic melanoma who had progressed after receiving PD-1 and PD-L1 suppression. The best overall response rate (ORR) to high-dose IL-2 was $22.5 \%$ for metastatic melanoma, with four complete responses and five partial responses. These findings encourage further investigation of high-dose IL-2 as an immunotherapy for metastatic melanoma patients. Moreover, the combination of immune checkpoint inhibitors and IL-2 therapy deserves further exploration. In addition, the exploration of targeting IL-2 is a critical complement to current immunotherapy and may help us better understand immunotherapy signal transduction (73).

\section{T-CELL AGONISTS TARGETING 4-1BB/OX40}

Immune checkpoint inhibitors can block cancer cells from escaping immune surveillance and improve $\mathrm{T}$ cell antitumor efficacy. Besides, antitumor T-cell functions are enhanced by targeting costimulatory molecules expressed on T-cell surfaces such as 4-1BB, OX40, inducible T-cell costimulator, and glucocorticoid-induced TNF receptor (GITR). 4-1BB (CD137) belonging to the TNF receptor family is expressed on a broad range of cell types, including activated $\mathrm{T}$ cells, dendritic cells (DCs), NK cells, B cells, monocytes, and neutrophils, and its activation caused by antibody ligation or 4-1 BB ligand contributes to T-cell activation (74). Utomilumab and urelumab, which are monoclonal antibody agonists (mAbAGs) targeting 4-1BB, have shown strong anti-tumor efficacy in preclinical models, but clinical studies have been limited by only marginal efficacy or serious liver toxicity. According to data from the trials (NCT00309023, NCT00612664, NCT01471210), urelumab monotherapy $(0.1 \mathrm{mg} / \mathrm{kg}$ every 3 weeks $)$ was well tolerated and immunoreactive, indicating that urelumab monotherapy and in combination with other immunooncology agents can be further assessed in patients with advanced solid tumors and lymphoma (75). Additionally, LVGN6051 is a novel 4-1BB mAb-AG, which improves the antitumor efficacy via regulating agonistic strength and Fc $\gamma \mathrm{R}$ affinity without liver damage (76).

The preclinical and clinical data indicated that melanoma treatment may move forward using agonists like OX40 (CD134, TNFRSF4), CD137, CD40, GITR, and CD27 activating costimulatory pathways (77). OX40 is a potent costimulatory protein in the tumor necrosis factor receptor superfamily, 
which actively participates in the positive regulation of CD4+ and $\mathrm{CD} 8+\mathrm{T}$ cell activity and inhibits the activity of regulatory $\mathrm{T}$ cells, showing an effective anti-tumor effect. In a phase I trial (NCT02315066), the safety and tolerability of ivuxolimab (an OX40 agonist monoclonal antibody) were assessed in patients with locally advanced or metastatic cancers. A partial response was obtained in three patients $(\mathrm{N}=52)$, and $56 \%$ of patients gained disease control. At 0.1 to $3.0 \mathrm{mg} / \mathrm{kg}$, elevated CD4+ central memory $\mathrm{T}$-cell proliferation and activation, as well as clonal expansion of CD4+ and CD8+ T cells, were seen in peripheral blood. An elevated OX40 expression and immune cell infiltration were observed in on-treatment tumor samples (78).

\section{POTENTIAL MECHANISMS OF IMMUNOTHERAPY RESISTANCE}

Even though checkpoint inhibitors have enhanced long-term survival, roughly $40 \%-50 \%$ of tumors are unresponsive to singleagent immune checkpoint inhibition, and these patients eventually develop drug resistance. Tumor-intrinsic resistance mechanisms to immune checkpoint inhibitor therapy included PD-L1 expression, mutational burden, neoantigen expression, epigenetic variations, type II interferon signaling, and antigen presentation pathways. In addition, tumor-extrinsic resistance mechanisms to immune checkpoint inhibitor therapy mainly included microbiome, PD-L1 expression on immune cells, tumoral and peripheral immune, and cell composition (79).

In the KEYNOTE-006 study, TMB, GEP, and PD-L1 were significantly associated with best overall response (BOR), progression-free survival (PFS), and overall survival (OS) in the pembrolizumab group. Patients with high TMB $(\geq 175)$ and GEP $(\geq-0.318)$ levels had a higher response rate to pembrolizumab treatment than those with low TMB and GEP levels (54\% vs. 14\%). Besides, patients with high TMB $(\geq 175)$ and PD-L1 MEL scores $(\geq 2)$ showed higher response rates to pembrolizumab treatment than those with low TMB and PD-L1 MEL scores (51\% vs. 33\%). However, only GEP exhibited substantial associations with PFS and OS in the ipilimumab group (80).

Patients with high TMB or high inflammatory signature score had a longer PFS and OS across all three treatment groups (nivolumab plus ipilimumab group, nivolumab group, and ipilimumab group) in the CheckMate 067 (NCT01844505) study. In the CheckMate 066 (NCT01721772) study, high TMB were significantly associated with PFS (HR 0.33; 95\% CI: $0.16,0.69 ; \mathrm{p}=0.0031$ ) and OS (HR 0.43; 95\% CI: 0.20, 0.91; $\mathrm{p}=0.027)$ in patients treated with nivolumab. Based on the results of the CheckMate 066 and CheckMate 067 studies, patients with high TMB and no BRAF mutation had longer survival. Besides, weak associations between PD-L1, TMB, and the inflammatory signature were found. A combination of TMB, inflammatory gene expression signature, and BRAF mutation status may predict response to immune checkpoint blockade in advanced melanoma $(81,82)$.
In comparison to patients with PFS $>12$ months, those with early PFS events ( $\leq 12$ months) had lower expression levels of known genes and gene expression signatures (GESs) associated with immune infiltration, such as interferon (IFN) $-\gamma$ and T cellinflamed in the COMBI-i trial. Lower tumor mutational burden (TMB) or T cell-inflamed GES levels, higher specific immunosuppressive TME signatures levels, and increased baseline circulating tumor DNA (ctDNA) levels were strongly associated with early PFS events. Biomarker profiles exhibited that patients with complete response (CR) had relatively low or undetectable baseline ctDNA levels. In subgroup analysis, the commonly used immunotherapy response biomarkers TMB and T cell-inflamed GES were not associated with CR, but patients who achieved CR had lower baseline levels of immunosuppressive TME signatures. When comparing baseline and 2 to 3 weeks of treatment, a rise in $\mathrm{T}$ cellinflamed GES was found independent of subsequent patient progression, while MAPK pathway activity scores (MPAS) and cell cycle GESs reduced from baseline to 2 to 3 weeks of treatment. Biopsy analysis of patients with early PFS events at 8 - to 12 -week biopsies exhibited a reduction in $\mathrm{T}$-cell inflammatory GES and an increase in MPAS. Correlative analysis between GES levels and tumor shrinkage following treatment with spartalizumab plus dabrafenib and trametinib exhibited that PI3K pathway GES levels were correlated with best overall tumor reduction, indicating that a compensatory signaling pathway for MAPK inhibition may be responsible for the lack of early response (55).

In the PIVOT-02 study (NCT02983045) study, the improved objective response rate and progression-free survival were observed in baseline tumor biopsies with high IFN- $\gamma$ GEP, high CD8+ TIL, high CD74, and high HLA-E. After treatment, CD4+ T cells were significantly upregulated, NK cell polyfunctionality was reduced, and polyfunctional CD8+ T cells were significantly enhanced only in patients with an objective response. The elevated polyfunctional response in $\mathrm{CD} 8+$ and CD4+ T cells seems to be promoted by the generation of cytokines with effector functions (57).

The mechanism of immunotherapy resistance mainly includes immune-desert or immune-cold tumor immunophenotypic models. In the immune-cold phenotype, stromal or vascular factors are responsible for the periphery of immune cells in the tumor microenvironment, leading to resistance to immunotherapy. In the immune-desert phenotype, the evidence of immune infiltrates in the tumor microenvironment is absent. In the immune-desert or immune-cold tumor, the number of regulatory cells entering the tumor microenvironment elevates, the secretion of immunosuppressive cytokines such as IL-8, TGF- $\beta$, VEGF elevate, and the expression of inhibitory receptors such as PD-1, CTLA-4, TIM3, LAG3, VISTA, BTLA, CD160 on T cells is enhanced. Combination therapy strategies that include immune checkpoint inhibitors may make the tumor microenvironment more immune-infiltrated, thus improving the effectiveness of immune checkpoint inhibitors (83). Melanoma cells with JAK1/2 deletion are resistant to IFN-induced anticancer activity, whereas melanoma cells with B2M loss block melanoma cells from being recognized by antigen-specific $\mathrm{T}$ cells and maybe therefore resistant 
to cytotoxicity. In vivo, intratumoral administration of Toll-like receptor 9 agonists and anti-PD-1 inhibitors can activate innate and adaptive immunity via natural killer (NK) and CD8 T cells, addressing JAK1/2 loss of resistance. The activation of NK-cell and CD4 T-cell using CD122-preferential interleukin (IL)-2 pathway agonists can address B2M-loss resistance. Stimulating NK cells and activating IFN signaling via pattern recognition receptors address the PD-1 inhibitor resistance caused by JAK1/2, and $\mathrm{B} 2 \mathrm{M}$ pathogenic mutations mediated by defective IFN receptor and antigen presentation pathways (84).

\section{CONCLUSIONS AND FUTURE PERSPECTIVES}

Pharmaceutical advancements have opened the path for precision cancer treatment in the present era of melanoma management. With the introduction of innovative therapeutic methods, such as BRAF and MEK inhibitors and immune checkpoint inhibitors, the prognosis of metastatic melanoma patients has dramatically enhanced in recent years. The optimal treatment strategy and medication sequence for patients with BRAF mutation or BRAF wild-type melanoma are still unclear. Most patients will only receive partial and short-term benefits from systemic therapy, and drug resistance and disease progression will almost inevitably occur. Combination therapies for different resistance mechanisms are a promising approach, but less toxic drug strategies are critical. Given the survival advantages in metastatic melanoma and the outcomes of the early-stage clinical trials, these treatment strategies can be

\section{REFERENCES}

1. Sung H, Ferlay J, Siegel RL, Laversanne M, Soerjomataram I, Jemal A, et al. Global Cancer Statistics 2020: GLOBOCAN Estimates of Incidence and Mortality Worldwide for 36 Cancers in 185 Countries. CA Cancer J Clin (2021) 71(3):209-49. doi: 10.3322/caac.21660

2. Siegel RL, Miller KD, Fuchs HE, Jemal A. Cancer Statistics, 2021. CA Cancer J Clin (2021) 71(1):7-33. doi: 10.3322/caac.21654

3. Leachman SA, Lucero OM, Sampson JE, Cassidy P, Bruno W, Queirolo P, et al. Identification, Genetic Testing, and Management of Hereditary Melanoma. Cancer Metastasis Rev (2017) 36(1):77-90. doi: 10.1007/s10555-017-9661-5

4. Toussi A, Mans N, Welborn J, Kiuru M. Germline Mutations Predisposing to Melanoma. J Cutan Pathol (2020) 47(7):606-16. doi: 10.1111/cup.13689

5. Carr S, Smith C, Wernberg J. Epidemiology and Risk Factors of Melanoma. Surg Clin North Am (2020) 100(1):1-12. doi: 10.1016/j.suc.2019.09.005

6. Bataille V. It's Not All Sunshine: non-Sun-Related Melanoma Risk-Factors. Acta Derm Venereol (2020) 100(11):adv00137. doi: 10.2340/00015555-3492

7. Rastrelli M, Tropea S, Rossi CR, Alaibac M. Melanoma: Epidemiology, Risk Factors, Pathogenesis, Diagnosis and Classification. In Vivo (2014) 28(6):1005-11.

8. Cancer Genome Atlas N. Genomic Classification of Cutaneous Melanoma. Cell (2015) 161(7):1681-96. doi: 10.1016/j.cell.2015.05.044

9. Hodis E, Watson IR, Kryukov GV, Arold ST, Imielinski M, Theurillat JP, et al. A Landscape of Driver Mutations in Melanoma. Cell (2012) 150(2):251-63. doi: 10.1016/j.cell.2012.06.024

10. Curti BD, Faries MB. Recent Advances in the Treatment of Melanoma. N Engl J Med (2021) 384(23):2229-40. doi: 10.1056/NEJMra2034861

11. Krauthammer M, Kong Y, Ha BH, Evans P, Bacchiocchi A, McCusker JP, et al. Exome Sequencing Identifies Recurrent Somatic RAC1 Mutations in Melanoma. Nat Genet (2012) 44(9):1006-14. doi: 10.1038/ng.2359 used as adjuvant treatments for high-risk resected stage III melanoma. For neoadjuvant therapy of high-risk stage III melanoma, these treatment strategies have also been explored in several studies, and preliminary data indicates that they might be a very promising strategy in this scenario. The current results still need to be confirmed by prospective clinical trials before they can be used in daily clinical practice. Ongoing clinical research can improve our understanding of melanoma, develop more effective and less toxic treatments, and improve the prognosis of patients with melanoma. Although immune checkpoint inhibitors have revolutionized melanoma patients, successful treatments for melanoma are still a long way off.

\section{AUTHOR CONTRIBUTIONS}

HHZ, FL, HRZ, and CZ designed the study and supervised it. HHZ and FL collected data. HHZ performed statistical analysis. HRZ interpreted data and drafted the manuscript. CZ contributed to administrative, technical and material support. All authors contributed to the article and approved the submitted version.

\section{FUNDING}

This present study was supported in part by the National Natural Science Foundation of China (grant numbers: 81660755), and the Science and Technology Project of Shenzhen of China (grant numbers: JCYJ20190808162605484).

12. Van Allen EM, Wagle N, Sucker A, Treacy DJ, Johannessen CM, Goetz EM, et al. The Genetic Landscape of Clinical Resistance to RAF Inhibition in Metastatic Melanoma. Cancer Discovery (2014) 4(1):94-109. doi: 10.1158/ 2159-8290.CD-13-0617

13. Randic T, Kozar I, Margue C, Utikal J, Kreis S. NRAS Mutant Melanoma: Towards Better Therapies. Cancer Treat Rev (2021) 99:102238. doi: 10.1016/ j.ctrv.2021.102238

14. Fedorenko IV, Gibney GT, Smalley KS. NRAS Mutant Melanoma: Biological Behavior and Future Strategies for Therapeutic Management. Oncogene (2013) 32(25):3009-18. doi: 10.1038/onc.2012.453

15. Hayward NK, Wilmott JS, Waddell N, Johansson PA, Field MA, Nones K, et al. Whole-Genome Landscapes of Major Melanoma Subtypes. Nature (2017) 545(7653):175-80. doi: 10.1038/nature22071

16. Conway JR, Dietlein F, Taylor-Weiner A, AlDubayan S, Vokes N, Keenan T, et al. Integrated Molecular Drivers Coordinate Biological and Clinical States in Melanoma. Nat Genet (2020) 52(12):1373-83. doi: 10.1038/s41588-020-00739-1

17. Ming Z, Lim SY, Rizos H. Genetic Alterations in the INK4a/ARF Locus: Effects on Melanoma Development and Progression. Biomolecules (2020) 10 (10):1447. doi: 10.3390/biom10101447

18. Zeng H, Jorapur A, Shain AH, Lang UE, Torres R, Zhang Y, et al. Bi-Allelic Loss of CDKN2A Initiates Melanoma Invasion via BRN2 Activation. Cancer Cell (2018) 34(1):56-68.e9. doi: 10.1016/j.ccell.2018.05.014

19. Lionarons DA, Hancock DC, Rana S, East P, Moore C, Murillo MM, et al. RAC1(P29S) Induces a Mesenchymal Phenotypic Switch via Serum Response Factor to Promote Melanoma Development and Therapy Resistance. Cancer Cell (2019) 36(1):68-83.e9. doi: 10.1016/j.ccell.2019.05.015

20. Chapman PB, Hauschild A, Robert C, Haanen JB, Ascierto P, Larkin J, et al. Improved Survival With Vemurafenib in Melanoma With BRAF V600E Mutation. N Engl J Med (2011) 364(26):2507-16. doi: 10.1056/NEJMoa1103782 
21. Robert C, Karaszewska B, Schachter J, Rutkowski P, Mackiewicz A, Stroiakovski D, et al. Improved Overall Survival in Melanoma With Combined Dabrafenib and Trametinib. N Engl J Med (2015) 372(1):30-9. doi: 10.1056/NEJMoa1412690

22. Long GV, Stroyakovskiy D, Gogas H, Levchenko E, de Braud F, Larkin J, et al. Dabrafenib and Trametinib Versus Dabrafenib and Placebo for Val600 BRAFMutant Melanoma: A Multicentre, Double-Blind, Phase 3 Randomised Controlled Trial. Lancet (2015) 386(9992):444-51. doi: 10.1016/S0140-6736 (15)60898-4

23. Robert C, Grob JJ, Stroyakovskiy D, Karaszewska B, Hauschild A, Levchenko E, et al. Five-Year Outcomes With Dabrafenib Plus Trametinib in Metastatic Melanoma. N Engl J Med (2019) 381(7):626-36. doi: 10.1056/NEJMoa1904059

24. Larkin J, Ascierto PA, Dreno B, Atkinson V, Liszkay G, Maio M, et al. Combined Vemurafenib and Cobimetinib in BRAF-Mutated Melanoma. N Engl J Med (2014) 371(20):1867-76. doi: 10.1056/NEJMoa1408868

25. Ascierto PA, McArthur GA, Dreno B, Atkinson V, Liszkay G, Di Giacomo AM, et al. Cobimetinib Combined With Vemurafenib in Advanced BRAF (V600)-Mutant Melanoma (Cobrim): Updated Efficacy Results From a Randomised, Double-Blind, Phase 3 Trial. Lancet Oncol (2016) 17(9):124860. doi: 10.1016/S1470-2045(16)30122-X

26. Ascierto PA, Dreno B, Larkin J, Ribas A, Liszkay G, Maio M, et al. 5-Year Outcomes With Cobimetinib Plus Vemurafenib in BRAF (V600) MutationPositive Advanced Melanoma: Extended Follow-Up of the Cobrim Study. Clin Cancer Res (2021) 27(19):5225-35. doi: 10.1158/1078-0432.CCR-21-0809

27. Dummer R, Ascierto PA, Gogas HJ, Arance A, Mandala M, Liszkay G, et al. Encorafenib Plus Binimetinib Versus Vemurafenib or Encorafenib in Patients With BRAF-Mutant Melanoma (COLUMBUS): A Multicentre, Open-Label, Randomised Phase 3 Trial. Lancet Oncol (2018) 19(5):603-15. doi: 10.1016/ S1470-2045(18)30142-6

28. Ascierto PA, Dummer R, Gogas HJ, Flaherty KT, Arance A, Mandala M, et al. Update on Tolerability and Overall Survival in COLUMBUS: Landmark Analysis of a Randomised Phase 3 Trial of Encorafenib Plus Binimetinib vs Vemurafenib or Encorafenib in Patients With BRAF V600-Mutant Melanoma. Eur J Cancer (2020) 126:33-44. doi: 10.1016/j.ejca.2019.11.016

29. Dummer R, Hauschild A, Santinami M, Atkinson V, Mandala M, Kirkwood JM, et al. Five-Year Analysis of Adjuvant Dabrafenib Plus Trametinib in Stage III Melanoma. N Engl J Med (2020) 383(12):1139-48. doi: 10.1056/NEJMoa2005493

30. Long GV, Hauschild A, Santinami M, Atkinson V, Mandala M, ChiarionSileni V, et al. Adjuvant Dabrafenib Plus Trametinib in Stage III BRAFMutated Melanoma. N Engl J Med (2017) 377(19):1813-23. doi: 10.1056/ NEJMoa1708539

31. Long GV, Saw RPM, Lo S, Nieweg OE, Shannon KF, Gonzalez M, et al. Neoadjuvant Dabrafenib Combined With Trametinib for Resectable, Stage IIIB-C, BRAF(V600) Mutation-Positive Melanoma (Neocombi): A SingleArm, Open-Label, Single-Centre, Phase 2 Trial. Lancet Oncol (2019) 20 (7):961-71. doi: 10.1016/S1470-2045(19)30331-6

32. Guo J, Si L, Kong Y, Flaherty KT, Xu X, Zhu Y, et al. Open-Label, Single-Arm Trial of Imatinib Mesylate in Patients With Metastatic Melanoma Harboring C-Kit Mutation or Amplification. J Clin Oncol (2011) 29(21):2904-9. doi: 10.1200/JCO.2010.33.9275

33. Carvajal RD. Another Option in Our KIT of Effective Therapies for Advanced Melanoma. J Clin Oncol (2013) 31(26):3173-5. doi: 10.1200/JCO.2013.50.3144

34. Bastian BC, Esteve-Puig R. Targeting Activated KIT Signaling for Melanoma Therapy. J Clin Oncol (2013) 31(26):3288-90. doi: 10.1200/JCO.2013.50.3227

35. Lyle M, Long GV. Diagnosis and Treatment of KIT-Mutant Metastatic Melanoma. J Clin Oncol (2013) 31(26):3176-81. doi: 10.1200/JCO.2013.50.4662

36. Dummer R, Schadendorf D, Ascierto PA, Arance A, Dutriaux C, Di Giacomo AM, et al. Binimetinib Versus Dacarbazine in Patients With Advanced NRASMutant Melanoma (NEMO): A Multicentre, Open-Label, Randomised, Phase 3 Trial. Lancet Oncol (2017) 18(4):435-45. doi: 10.1016/S1470-2045(17)30180-8

37. Long GV, Fung C, Menzies AM, Pupo GM, Carlino MS, Hyman J, et al. Increased MAPK Reactivation in Early Resistance to Dabrafenib/Trametinib Combination Therapy of BRAF-Mutant Metastatic Melanoma. Nat Commun (2014) 5:5694. doi: 10.1038/ncomms6694

38. Ojha R, Leli NM, Onorati A, Piao S, Verginadis II, Tameire F, et al. ER Translocation of the MAPK Pathway Drives Therapy Resistance in BRAFMutant Melanoma. Cancer Discovery (2019) 9(3):396-415. doi: 10.1158/21598290.CD-18-0348
39. Lu H, Liu S, Zhang G, Bin W, Zhu Y, Frederick DT, et al. PAK Signalling Drives Acquired Drug Resistance to MAPK Inhibitors in BRAF-Mutant Melanomas. Nature (2017) 550(7674):133-6. doi: 10.1038/nature24040

40. Yen I, Shanahan F, Lee J, Hong YS, Shin SJ, Moore AR, et al. ARAF Mutations Confer Resistance to the RAF Inhibitor Belvarafenib in Melanoma. Nature (2021) 594(7863):418-23. doi: 10.1038/s41586-021-03515-1

41. Ablain J, Liu S, Moriceau G, Lo RS, Zon LI. SPRED1 Deletion Confers Resistance to MAPK Inhibition in Melanoma. J Exp Med (2021) 218(3): e20201097. doi: 10.1084/jem.20201097

42. Teh JLF, Cheng PF, Purwin TJ, Nikbakht N, Patel P, Chervoneva I, et al. In Vivo E2F Reporting Reveals Efficacious Schedules of MEK1/2-CDK4/6 Targeting and Mtor-S6 Resistance Mechanisms. Cancer Discovery (2018) 8 (5):568-81. doi: 10.1158/2159-8290.CD-17-0699

43. Romano G, Chen PL, Song P, McQuade JL, Liang RJ, Liu M, et al. A Preexisting Rare PIK3CA(E545K) Subpopulation Confers Clinical Resistance to MEK Plus CDK4/6 Inhibition in NRAS Melanoma and Is Dependent on S6K1 Signaling. Cancer Discovery (2018) 8(5):556-67. doi: 10.1158/2159-8290.CD-17-0745

44. Matter AV, Micaletto S, Urner-Bloch U, Dummer R, Goldinger SM. Long-Term Response to Intermittent Binimetinib in Patients With NRAS-Mutant Melanoma. Oncologist (2020) 25(11):e1593-e7. doi: 10.1634/theoncologist.2019-0656

45. Dummer R, Brase JC, Garrett J, Campbell CD, Gasal E, Squires M, et al. Adjuvant Dabrafenib Plus Trametinib Versus Placebo in Patients With Resected, BRAF(V600)-Mutant, Stage III Melanoma (COMBI-AD): Exploratory Biomarker Analyses From a Randomised, Phase 3 Trial. Lancet Oncol (2020) 21(3):358-72. doi: 10.1016/S1470-2045(20)30062-0

46. Yan Y, Wongchenko MJ, Robert C, Larkin J, Ascierto PA, Dreno B, et al. Genomic Features of Exceptional Response in Vemurafenib +/- CobimetinibTreated Patients With BRAF (V600)-Mutated Metastatic Melanoma. Clin Cancer Res (2019) 25(11):3239-46. doi: 10.1158/1078-0432.CCR-18-0720

47. Puzanov I, Ribas A, Robert C, Schachter J, Nyakas M, Daud A, et al. Association of BRAF V600E/K Mutation Status and Prior BRAF/MEK Inhibition With Pembrolizumab Outcomes in Advanced Melanoma: Pooled Analysis of 3 Clinical Trials. JAMA Oncol (2020) 6(8):1256-64. doi: 10.1001/jamaoncol.2020.2288

48. Hamid O, Puzanov I, Dummer R, Schachter J, Daud A, Schadendorf D, et al. Final Analysis of a Randomised Trial Comparing Pembrolizumab Versus Investigator-Choice Chemotherapy for Ipilimumab-Refractory Advanced Melanoma. Eur J Cancer (2017) 86:37-45. doi: 10.1016/j.ejca.2017.07.022

49. Robert C, Ribas A, Schachter J, Arance A, Grob JJ, Mortier L, et al. Pembrolizumab Versus Ipilimumab in Advanced Melanoma (KEYNOTE006): Post-Hoc 5-Year Results From an Open-Label, Multicentre, Randomised, Controlled, Phase 3 Study. Lancet Oncol (2019) 20(9):123951. doi: 10.1016/S1470-2045(19)30388-2

50. Larkin J, Minor D, D’Angelo S, Neyns B, Smylie M, Miller WH Jr, et al. Overall Survival in Patients With Advanced Melanoma Who Received Nivolumab Versus Investigator's Choice Chemotherapy in Checkmate 037: A Randomized, Controlled, Open-Label Phase III Trial. J Clin Oncol (2018) 36 (4):383-90. doi: 10.1200/JCO.2016.71.8023

51. Robert C, Long GV, Brady B, Dutriaux C, Di Giacomo AM, Mortier L, et al. Five-Year Outcomes With Nivolumab in Patients With Wild-Type BRAF Advanced Melanoma. J Clin Oncol (2020) 38(33):3937-46. doi: 10.1200/ JCO.20.00995

52. Pires da Silva I, Ahmed T, Reijers ILM, Weppler AM, Betof Warner A, Patrinely JR, et al. Ipilimumab Alone or Ipilimumab Plus Anti-PD-1 Therapy in Patients With Metastatic Melanoma Resistant to Anti-PD-(L)1 Monotherapy: A Multicentre, Retrospective, Cohort Study. Lancet Oncol (2021) 22(6):836-47. doi: 10.1016/S1470-2045(21)00097-8

53. Larkin J, Chiarion-Sileni V, Gonzalez R, Grob JJ, Rutkowski P, Lao CD, et al. FiveYear Survival With Combined Nivolumab and Ipilimumab in Advanced Melanoma. N Engl J Med (2019) 381(16):1535-46. doi: 10.1056/NEJMoa1910836

54. Wang Y, Liu S, Yang Z, Algazi AP, Lomeli SH, Wang Y, et al. Anti-PD-1/L1 Lead-in Before MAPK Inhibitor Combination Maximizes Antitumor Immunity and Efficacy. Cancer Cell (2021) 39(10):1375-87. doi: 10.1016/ j.ccell.2021.07.023

55. Dummer R, Lebbe C, Atkinson V, Mandala M, Nathan PD, Arance A, et al. Combined PD-1, BRAF and MEK Inhibition in Advanced BRAF-Mutant Melanoma: Safety Run-in and Biomarker Cohorts of COMBI-I. Nat Med (2020) 26(10):1557-63. doi: 10.1038/s41591-020-1082-2 
56. Gutzmer R, Stroyakovskiy D, Gogas H, Robert C, Lewis K, Protsenko S, et al. Atezolizumab, Vemurafenib, and Cobimetinib as First-Line Treatment for Unresectable Advanced BRAF(V600) Mutation-Positive Melanoma (Imspire150): Primary Analysis of the Randomised, Double-Blind, PlaceboControlled, Phase 3 Trial. Lancet (2020) 395(10240):1835-44. doi: 10.1016/ S0140-6736(20)30934-X

57. Diab A, Tykodi SS, Daniels GA, Maio M, Curti BD, Lewis KD, et al. Bempegaldesleukin Plus Nivolumab in First-Line Metastatic Melanoma. J Clin Oncol (2021) 39(26):2914-25. doi: 10.1200/JCO.21.00675

58. Eggermont AMM, Blank CU, Mandala M, Long GV, Atkinson VG, Dalle S, et al. Adjuvant Pembrolizumab Versus Placebo in Resected Stage III Melanoma (EORTC 1325-MG/KEYNOTE-054): Distant Metastasis-Free Survival Results From a Double-Blind, Randomised, Controlled, Phase 3 Trial. Lancet Oncol (2021) 22(5):643-54. doi: 10.1016/S1470-2045(21)00065-6

59. Eggermont AMM, Kicinski M, Blank CU, Mandala M, Long GV, Atkinson V, et al. Association Between Immune-Related Adverse Events and Recurrence-Free Survival Among Patients With Stage III Melanoma Randomized to Receive Pembrolizumab or Placebo: A Secondary Analysis of a Randomized Clinical Trial. JAMA Oncol (2020) 6(4):519-27. doi: 10.1001/jamaoncol.2019.5570

60. Luke JJ, Ascierto PA, Carlino MS, Gershenwald JE, Grob JJ, Hauschild A, et al. KEYNOTE-716: Phase III Study of Adjuvant Pembrolizumab Versus Placebo in Resected High-Risk Stage II Melanoma. Future Oncol (2020) 16(3):442938. doi: 10.2217/fon-2019-0666

61. Eggermont AMM, Blank CU, Mandala M, Long GV, Atkinson VG, Dalle S, et al. Longer Follow-Up Confirms Recurrence-Free Survival Benefit of Adjuvant Pembrolizumab in High-Risk Stage III Melanoma: Updated Results From the EORTC 1325-MG/KEYNOTE-054 Trial. J Clin Oncol (2020) 38(33):3925-36. doi: 10.1200/JCO.20.02110

62. Tarhini AA, Lee SJ, Hodi FS, Rao UNM, Cohen GI, Hamid O, et al. Phase III Study of Adjuvant Ipilimumab ( 3 or $10 \mathrm{Mg} / \mathrm{Kg}$ ) Versus High-Dose Interferon Alfa-2b for Resected High-Risk Melanoma: North American Intergroup E1609. J Clin Oncol (2020) 38(6):567-75. doi: 10.1200/JCO.19.01381

63. Ascierto PA, Del Vecchio M, Mandala M, Gogas H, Arance AM, Dalle S, et al. Adjuvant Nivolumab Versus Ipilimumab in Resected Stage IIIB-C and Stage IV Melanoma (Checkmate 238): 4-Year Results From a Multicentre, DoubleBlind, Randomised, Controlled, Phase 3 Trial. Lancet Oncol (2020) 21 (11):1465-77. doi: 10.1016/S1470-2045(20)30494-0

64. Zimmer L, Livingstone E, Hassel JC, Fluck M, Eigentler T, Loquai C, et al. Adjuvant Nivolumab Plus Ipilimumab or Nivolumab Monotherapy Versus Placebo in Patients With Resected Stage IV Melanoma With No Evidence of Disease (IMMUNED): A Randomised, Double-Blind, Placebo-Controlled, Phase 2 Trial. Lancet (2020) 395(10236):1558-68. doi: 10.1016/S0140-6736 (20)30417-7

65. Rozeman EA, Hoefsmit EP, Reijers ILM, Saw RPM, Versluis JM, Krijgsman O, et al. Survival and Biomarker Analyses From the Opacin-Neo and Opacin Neoadjuvant Immunotherapy Trials in Stage III Melanoma. Nat Med (2021) 27(2):256-63. doi: 10.1038/s41591-020-01211-7

66. Menzies AM, Amaria RN, Rozeman EA, Huang AC, Tetzlaff MT, van de Wiel BA, et al. Pathological Response and Survival With Neoadjuvant Therapy in Melanoma: A Pooled Analysis From the International Neoadjuvant Melanoma Consortium (INMC). Nat Med (2021) 27(2):301-9. doi: 10.1038/s41591-020-01188-3

67. Borgers JSW, Haanen J. Cellular Therapy and Cytokine Treatments for Melanoma. Hematol Oncol Clin North Am (2021) 35(1):129-44. doi: 10.1016/ j.hoc. 2020.08 .014

68. Sarnaik AA, Hamid O, Khushalani NI, Lewis KD, Medina T, Kluger HM, et al. Lifileucel, a Tumor-Infiltrating Lymphocyte Therapy, in Metastatic Melanoma. J Clin Oncol (2021) 39(24):2656-66. doi: 10.1200/JCO.21.00612

69. Kverneland AH, Chamberlain CA, Borch TH, Nielsen M, Mork SK, Kjeldsen JW, et al. Adoptive Cell Therapy With Tumor-Infiltrating Lymphocytes Supported by Checkpoint Inhibition Across Multiple Solid Cancer Types. J Immunother Cancer (2021) 9(10):e003499. doi: 10.1136/jitc-2021-003499

70. Simon B, Uslu U. CAR-T Cell Therapy in Melanoma: A Future Success Story? Exp Dermatol (2018) 27(12):1315-21. doi: 10.1111/exd.13792

71. Rosenberg SA, Yang JC, Topalian SL, Schwartzentruber DJ, Weber JS, Parkinson DR, et al. Treatment of 283 Consecutive Patients With Metastatic Melanoma or Renal Cell Cancer Using High-Dose Bolus Interleukin 2. JAMA (1994) 271 (12):907-13. doi: 10.1001/jama.1994.03510360033032
72. Atkins MB, Lotze MT, Dutcher JP, Fisher RI, Weiss G, Margolin K, et al. High-Dose Recombinant Interleukin 2 Therapy for Patients With Metastatic Melanoma: Analysis of 270 Patients Treated Between 1985 and 1993. J Clin Oncol (1999) 17(7):2105-16. doi: 10.1200/JCO.1999.17.7.2105

73. Buchbinder EI, Dutcher JP, Daniels GA, Curti BD, Patel SP, Holtan SG, et al. Therapy With High-Dose Interleukin-2 (HD IL-2) in Metastatic Melanoma and Renal Cell Carcinoma Following PD1 or PDL1 Inhibition. J Immunother Cancer (2019) 7(1):49. doi: 10.1186/s40425-019-0522-3

74. Chester C, Sanmamed MF, Wang J, Melero I. Immunotherapy Targeting 41BB: Mechanistic Rationale, Clinical Results, and Future Strategies. Blood (2018) 131(1):49-57. doi: 10.1182/blood-2017-06-741041

75. Segal NH, Logan TF, Hodi FS, McDermott D, Melero I, Hamid O, et al. Results From an Integrated Safety Analysis of Urelumab, an Agonist AntiCD137 Monoclonal Antibody. Clin Cancer Res (2017) 23(8):1929-36. doi: 10.1158/1078-0432.CCR-16-1272

76. Qi X, Li F, Wu Y, Cheng C, Han P, Wang J, et al. Optimization of 4-1BB Antibody for Cancer Immunotherapy by Balancing Agonistic Strength With Fcgammar Affinity. Nat Commun (2019) 10(1):2141. doi: 10.1038/s41467019-10088-1

77. He S, Xu J, Wu J. The Emerging Role of Co-Stimulatory Molecules and Their Agonistic Mab-Based Combination Therapies in Melanoma. Int Immunopharmacol (2020) 89(Pt B):107097. doi: 10.1016/j.intimp.2020.107097

78. Diab A, Hamid O, Thompson JA, Ros W, Eskens F, Doi T, et al. Open-Label, Dose-Escalation Study of the OX40 Agonist Ivuxolimab in Patients With Locally Advanced or Metastatic Cancers. Clin Cancer Res (2021). doi: 10.1158/ 1078-0432.CCR-21-0845

79. Bagchi S, Yuan R, Engleman EG. Immune Checkpoint Inhibitors for the Treatment of Cancer: Clinical Impact and Mechanisms of Response and Resistance. Annu Rev Pathol (2021) 16:223-49. doi: 10.1146/annurev-pathol-042020-042741

80. Ribas A, Robert C, Schachter J, Long GV, Arance A, Carlino MS, et al. Abstract 4217: Tumor Mutational Burden (TMB), T Cell-Inflamed Gene Expression Profile (GEP) and PD-L1 Are Independently Associated With Response to Pembrolizumab (Pembro) in Patients With Advanced Melanoma in the KEYNOTE (KN)-006 Study. Cancer Res (2019) 79(13 Supplement):4217. doi: 10.1158/1538-7445.Am2019-4217

81. Hodi FS, Wolchok JD, Schadendorf D, Larkin J, Qian M, Saci A, et al. Abstract CT037: Genomic Analyses and Immunotherapy in Advanced Melanoma. Cancer Res (2019) 79(13 Supplement):CT037-CT. doi: 10.1158/15387445.Am2019-ct037

82. Hodi FS, Wolchok JD, Schadendorf D, Larkin J, Long GV, Qian X, et al. TMB and Inflammatory Gene Expression Associated With Clinical Outcomes Following Immunotherapy in Advanced Melanoma. Cancer Immunol Res (2021) 9(10):1202-13. doi: 10.1158/2326-6066.CIR-20-0983

83. Trojaniello C, Vitale MG, Scarpato L, Esposito A, Ascierto PA. Melanoma Immunotherapy: Strategies to Overcome Pharmacological Resistance. Expert Rev Anticancer Ther (2020) 20(4):289-304. doi: 10.1080/14737140.2020.1745634

84. Torrejon DY, Abril-Rodriguez G, Champhekar AS, Tsoi J, Campbell KM, Kalbasi A, et al. Overcoming Genetically Based Resistance Mechanisms to PD1 Blockade. Cancer Discovery (2020) 10(8):1140-57. doi: 10.1158/2159-8290. CD-19-1409

Conflict of Interest: The authors declare that the research was conducted in the absence of any commercial or financial relationships that could be construed as a potential conflict of interest.

Publisher's Note: All claims expressed in this article are solely those of the authors and do not necessarily represent those of their affiliated organizations, or those of the publisher, the editors and the reviewers. Any product that may be evaluated in this article, or claim that may be made by its manufacturer, is not guaranteed or endorsed by the publisher.

Copyright $\odot 2021$ Zeng, Liu, Zhou and Zeng. This is an open-access article distributed under the terms of the Creative Commons Attribution License (CC BY). The use, distribution or reproduction in other forums is permitted, provided the original author(s) and the copyright owner(s) are credited and that the original publication in this journal is cited, in accordance with accepted academic practice. No use, distribution or reproduction is permitted which does not comply with these terms. 